\title{
The MEKASPEK project - a new step towards the utmost photometric accuracy
}

\author{
K. H. Mantel, H. Barwig, S. Kiesewetter \\ Universitaets-Sternwarte Muenchen, Fed. Rep. Germany
}

\begin{abstract}
We present the design of a new 4 channel, fiberoptic spectrophotometer which allows to measure the object, two comparison stars and sky background simultaneously throughout the whole optical wavelength range with high efficiency. The overall spectral resolution of about 50 in combination with oversampling allows to eliminate colour dependent extinction effects to a high degree and guarantees accurate transformations to other broad band photometric systems.
\end{abstract}

\section{Introduction}

Since the first eye estimated photometry by Ptolemaeus and Hipparchos, about 2000 years ago, photometric accuracy has increased from half a magnitude to some millimagnitudes. However, in spite of the enormous instrumental efforts that have been made after the adoption of photoelectric detectors in order to push forward the limits of accuracy, no further significant improvement could be achieved.

A careful reexamination and discussion of the whole measuring process and the reduction procedure by Young $(1974,1988)$ revealed that the low precission achieved so far, was caused by an insufficiant treatment of higher order terms within the theory of extinction correction and colour transformation. Young (1988) showed that even within the classical Stroemgren-King-Theory, second order terms can be treated correctly provided that the filtercurves fulfil the requirements of the sampling theorem (Shannon 1949).

Four years ago, the project MEKASPEK ('Multi Channel Spectral Photometer') was initiated at the Universitaets-Sternwarte Muenchen (Mantel et al. 1988). The major aim of this project was to develop a new 4-channel spectral photometer in accordance with the requirements of the sampling theorem which would be able to achieve the utmost accuracy reachable with today's technology. This paper discusses the main features of this instruments. 


\section{Instrumental Design}

Within the MEKASPEK project a new photometer concept has been developed that may be characterized by the following features:

- high quantum efficiency throughout the whole optical wavelength range in order to keep the photon noise as small as possible

- simultaneous measurements of the object, two comparison stars and sky background for successful elimination of neutral atmospheric extinction effects

- spectral resolution in combination with oversampling allows to eliminate colour depended atmospheric effects and guarantees an accurate transformation to other broad band photometric systems

- optimized adaption of the entrance diaphragm's diameter to the actual seeing conditions

- high time resolution up to $10 \mathrm{~ms}$ in order to study rapid variable objects

The implementation of these features has been achieved by using four optical fibers which can be centered on the stars in the focal plane of the telescope.

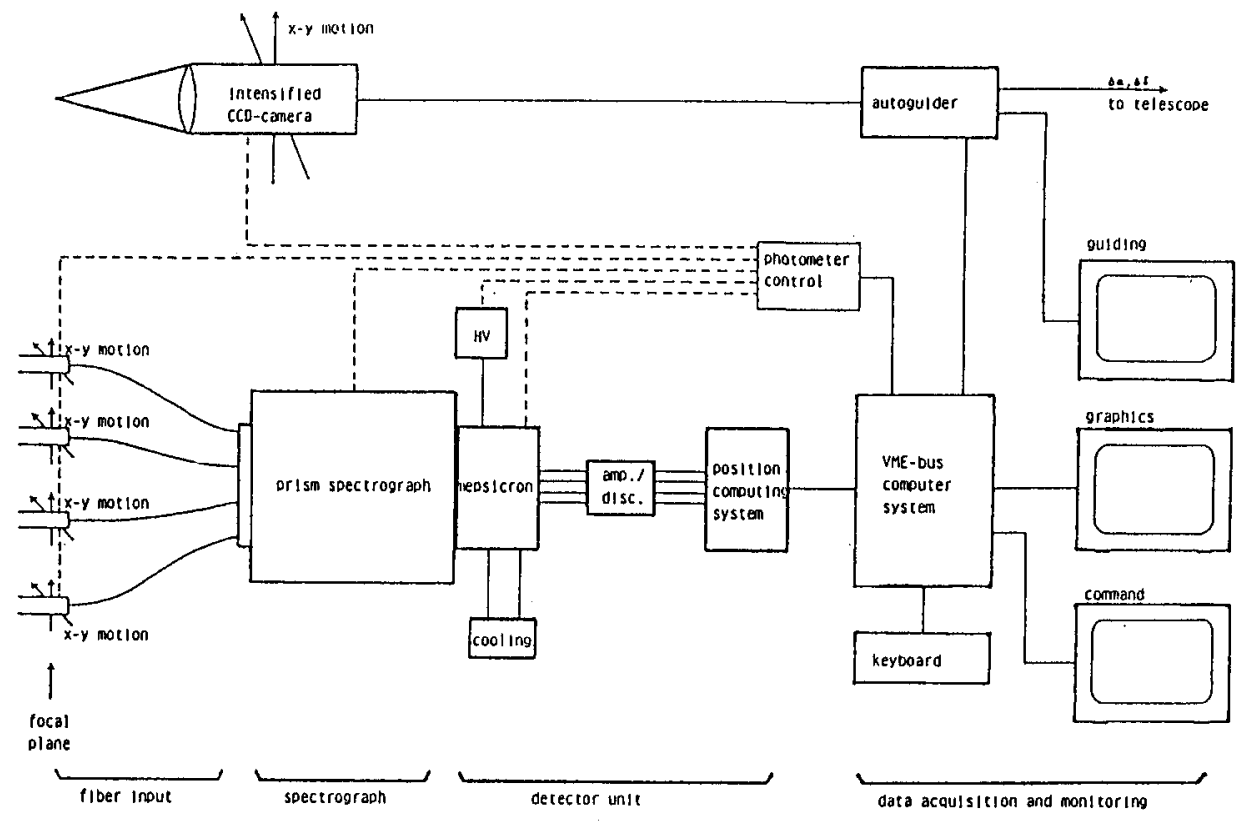

Figure 1: Block diagram of the 4-Channel-Spectral-Photometer. 
The fiber bundles fed the slit of a two beam prism spectrograph which has been optimized to reach high transmission throughout the whole optical wavelength region. The four spectra are focussed onto the surface of a two dimensional photon counting detector (MEPSICRON). The images can be registered with a time resolution of up to $10 \mathrm{~ms}$ by a VME-bus based computer system. Figure 1 shows a block diagram of the instrument.

\subsection{Optical fiber input channels}

Light transfer from the telescope focus to the spectrograph is achieved by fiber bundles. Each fiber bundle has a diameter of $800 \mu \mathrm{m}$ and consists of 130 single quartz fibers with a diameter of $70 \mu \mathrm{m}$ each which transform the circular entrance diaphragm to a rectangular slit shape. A fabry lens with a focal length of $6 \mathrm{~mm}$ projects the entrance pupil of the telescope onto the entrance of the fiber bundle, thus transforming spatial motion to angular variations. The varying $f$-number at the entrance of the fiber bundle is smeared out by degradation effects yielding $\mathrm{f}$ number variations of $f / 4.4$ to $f / 2.5$ at the entrance slit of the spectrograph. Each fiber bundle is equipped with an entrance diaphragm which may be exchanged under computer control and may vary between $0.6 \mathrm{~mm}$ and $2 \mathrm{~mm}$ in diameter.

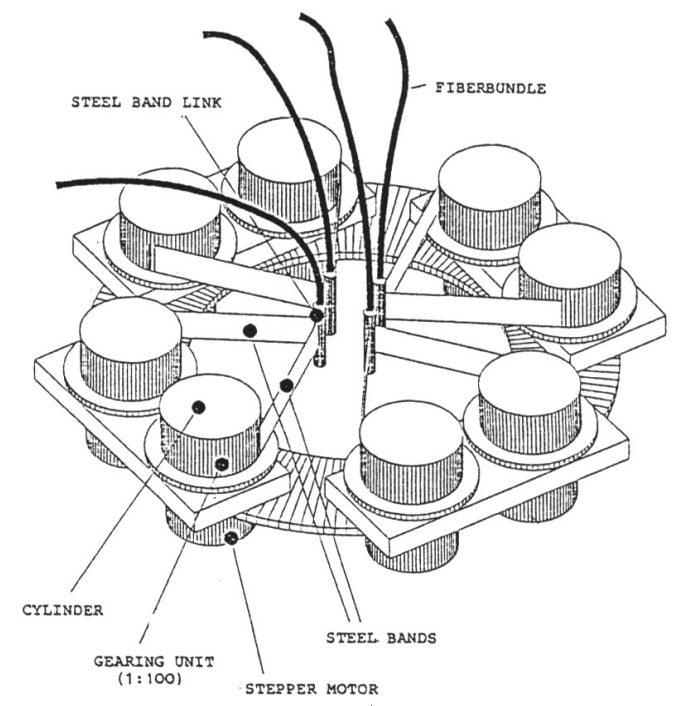

Figure 2: Fiber positioning unit.

Each fiber bundle is attached to two steel bands which are winded onto two steel drums (see Fig. 2). By rotating the drums via stepper motors, the length of 
the steel bands can be changed and thereby the positon of the fiber bundle in the focal plane. Test measurements have proved, that an positional accuracy of $10 \mu \mathrm{m}$ can be achieved.

\subsection{Spectrograph Unit and Detector}

In order to get a uniform spectral resolution throughout the whole optical wavelenght range a double beam prism spectrograph is used. The light from the four fiber bundles is split into two optical wavelength paths by use of a dichroitic beamsplitter. The two wavelength paths cover the spectral regions of 3400-5000 $\AA$ and $5000-9000 \AA$. Each path is dispersed seperatly by use of a prism, yielding a spectral resolution of about 50 (see Fig. 3). The FWHM of a monochromatic image covers about 7 pixels on the detector surface. The overall transmission of the spectrograph is about $65 \%$.

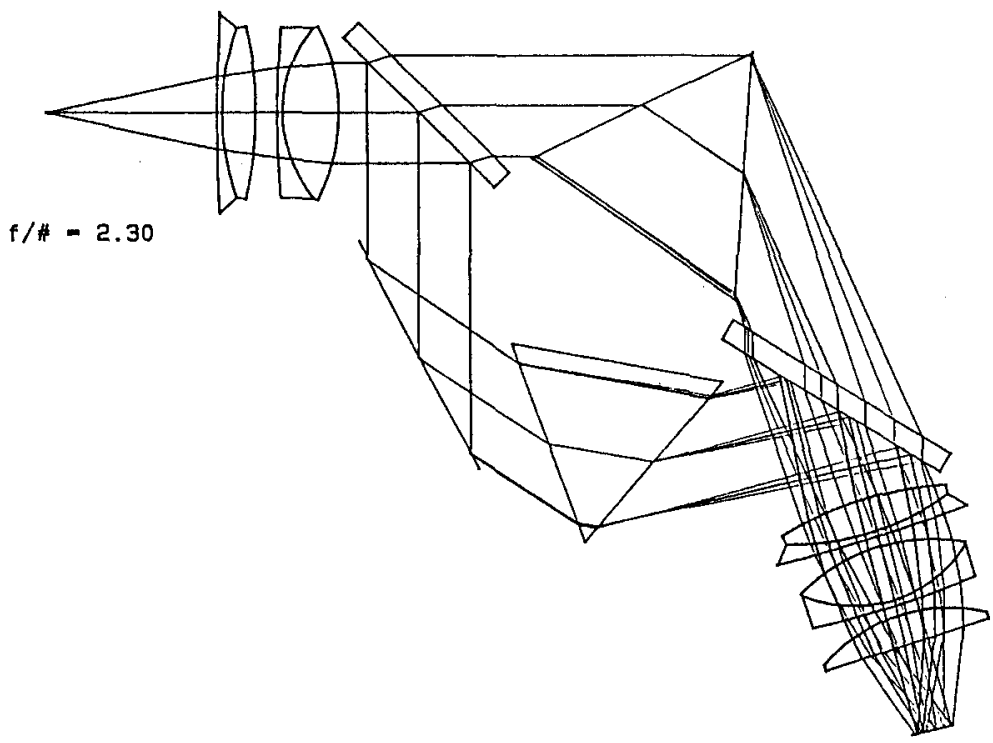

Figure 3: Double beam prism spectrograph.

The four spectra are recorded by a two dimensional photon counting resistive anode detector (MEPSICRON, Firmani et al. 1983). It has a sensitive area of $512 \times 512$ pixels and is equipped with a multialkali photocathode with a typical quantum efficiency fo $15-20 \%$ at $5000 \AA$. Five cascaded microchannel plates behind the photocathode accelarate the electrons to a resistive anode inducing a distinct charge distribution for each registered photon. The position of this distribution is determined by a positon computing system and send via an optical fiber transmitter to 
the host computer system. The detector is thermoelectrically cooled to $-25^{\circ} \mathrm{C}$. In comparison to a CCD this new detector has the following advantages:

- no readout noise

- dark current less than $50 \mathrm{cts} / \mathrm{s}$ or 1 count/pixel every 4 hours

- no overflow due to cosmic events

- time resolution of up to $1 \mathrm{~ms}$

- high frequency flat field variations less than $1 \%$

However care must be taken not to exceed the maximum count rate which is limited to $1 \times 10^{5} \mathrm{cts} / \mathrm{s}$ on the whole detector surface.

\section{Measurement Process and Data Reduction}

\subsection{Online Reduction}

For each object a blue and a red spectrum is generated which extends over about 10 pixel rows on the detector surface. In order to reduce the data stream, only the exposed rows of the detector are summed up during an online reduction process. However, due to imaging errors the spectral resolution may vary between different pixel rows. This variation is corrected by means of software.

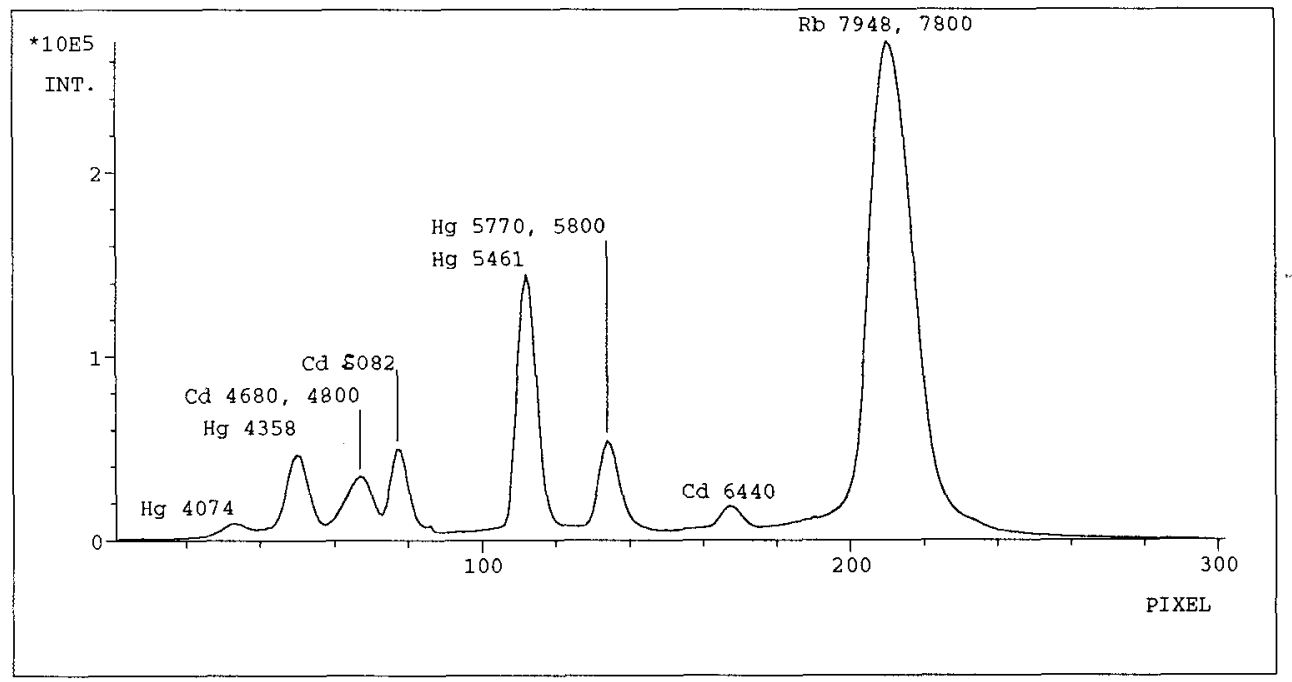

Figure 4: $\mathrm{Hg}$-Cd-Rb calibration spectrum.

From a calibration spectrum of $\mathrm{Hg}$-Cd-Rb lamps (see Fig. 4) the differential shift in wavelengths for each row and column on the detector is computed and stored in 
a transformation table. During the measurement process, this information is used to convert the individual spectrum to identical spectral resolution and dispersion throughout the 4 different input channels. This online reduction process diminishes the amount of data to be stored by about a factor of 200 and allows to achieve a maximum time resolution of about $10 \mathrm{~ms}$.

\subsection{Data Reduction}

As a result of the online reduction of the measurement data, four one dimensional spectra covering 300 pixels are obtained.

From identification of single lines in the Hg-Cd-Rb calibration spectrum a transformation between pixel and wavelength can be determined and the pixel scale can be converted to a lambda scale. Figure 4 shows the results of the registration of a $\mathrm{Hg}$-Cd-Rb calibration lamp.

As a monochromatic image covers 7 pixels on the detector surface, the data will be compressed by means of software to filtercurves with a FWHM of 7 pixels. The shift between succesive filtercurves is set up in order to obtain about 50 overlapping filtercurves which fulfil the sampling theorem (Shannon, 1949). The data will then be reduced to extraterrestic values by the standard second order Stroemgren-King Theorie as described by Young (1988). The overlapping filtercurves will guarantee a proper description of the second order moments required by the theory and will allow to reduce extinction effects properly. Furthermore the sampling theorem will also allow to transform the measured data with high accuracy to any broadband photometric system, which does not exceed the spectral resolution of the measured data.

This research program is partly supported by the Deutsche Forschungsgemeinschaft (grant BA 867/2-2).

\section{References:}

Firmani, C., Gutierrez, L., Ruiz, E., Bisiacchi, G.F., Salas, L., Paresce, F., 1983, in: SPIE Instrumentation in Astronomy V, 445, p. 192.

Mantel, K.H., Barwig, H., Kiesewetter, S., 1988, in: Proc. of New Directions in Spectrophotometry, Philip,A.G., Adelman,S.J., Hayes,D.S., (eds.), L. Davis Press.

Shannon, C.E., 1949: Proc. Inst. Radio Eng., 37, p. 10.

Young, A.T., 1974, in: Methods of Experimental Physics, Carleton,N., (ed.), Academic Press New York, Vol. 12, part A, p. 123.

Young, A.T., 1988, in: Proc. of the second Workshop on Improvements to Photometry, eds. W.J. Borucki, NASA Conference Pub. 10015, p. 215. 


\section{Discussion}

R.M. Redfern: I presume that the maximum count rate of $2.10^{5} \mathrm{c} / \mathrm{s}$ applies to a completely uniform illumination. Is there a problem with local deadtime?

Mantel: The maximum count rate per pixel is about $3.10^{4} \mathrm{c} / \mathrm{s}$ which is only a factor of ten below the integral maximum count rate. Therefore there is no problem with local deadtime.

J. Baruch: Have you thought about the problem of modal drift in the optical fibres? Unless steps are taken to ensure no movement of the fibres, and no temperature changes, modal drift can produce a few percent change in the apparent light transmission for very small movement and a temperature change of $5^{\circ} \mathrm{C}$. Movement of fibres in the focal plane will change the transmission and relative transmission of the bundles.

Mantel: During observations, the fibres are fixed, so there will be no change in transmission due to bending variations of the fibres. Besides that, test measurements have shown that $f$ numbers between $f / 30$ and $f / 2.7$ are smeared out by fibre degradation effects yielding variations of the light cone at the spectrograph entrance between $f / 4.4$ and $f / 2.5$. Therefore the spectrograph has been set up for an $f$ number of $f / 2.3$. So, even if temperature variations would change the light cone slightly, I do not expect any problems.

A. T. Young: I was worried by your remark about adjusting the entrance diaphragm to match the seeing. In the Proceedings of the (first) Workshop on Improvements to Photometry, Charles Ken Knight had an interesting paper on the wings of the star image, which contains a wavelength-dependent and large fraction of the stellar energy. Changing the diaphragm changes your photometric system.

Mantel: I'm aware of the problems that might be caused by the large wings of the stellar image. Nevertheless it is important to adapt the size of the entrance diaphragm to the focal-scale of the telescope and to the seeing condition in order to optimize the signal to noise ratio.

E. O'Mongain: At UCD we were responsible for the Hipparchos photometric calibration plan development, but now are involved in a similar transition in Remote Sensing from discrete band radiometry to low resolution spectral radiometry. Rather than reducing your data to discrete bands, have you considered using singular value decomposition methods to determine star spectral type and brightness directly? In this case extinction effects will have their own spectral characteristics and can be discriminated against.

Mantel: We are interested in accurate broad band photometry, therefore the data will be binned to widely overlapping filter curves. But depending on the scientific problem, it is also possible to use the whole spectrum, e.g. for determination of the spectral types.

J. Tinbergen: You seem to depend for passband wavelength stability on the stability of the spectrograph and detector. Do you monitor the wavelength stability, and if so, how?

K.H. Mantel: Prior to and after each observing run we do extensive calibration measurements with HgCdRb-line lamps. From these measurements the transformation between pixel and wavelengths, as well as the wavelength stability, can be determined. 